\title{
Selective Attention in an Insect Auditory Neuron
}

\author{
Gerald S. Pollack \\ Department of Biology, McGill University, Montreal, Quebec, Canada H3A 1 B1
}

Previous work (Pollack, 1986) showed that an identified auditory neuron of crickets, the omega neuron, selectively encodes the temporal structure of an ipsilateral sound stimulus when a contralateral stimulus is presented simultaneously, even though the contralateral stimulus is clearly encoded when it is presented alone. The present paper investigates the physiological basis for this selective response.

The selectivity for the ipsilateral stimulus is a result of the apparent intensity difference of ipsi- and contralateral stimuli, which is imposed by auditory directionality; when simultaneous presentation of stimuli from the 2 sides is mimicked by presenting low- and high-intensity stimuli simultaneously from the ipsilateral side, the neuron responds selectively to the high-intensity stimulus, even though the low-intensity stimulus is effective when it is presented alone. The selective encoding of the more intense (= ipsilateral) stimulus is due to intensity-dependent inhibition, which is superimposed on the cell's excitatory response to sound. Because of the inhibition, the stimulus with lower intensity (i.e., the contralateral stimulus) is rendered subthreshold, while the stimulus with higher intensity (the ipsilateral stimulus) remains above threshold. Consequently, the temporal structure of the low-intensity stimulus is filtered out of the neuron's spike train.

The source of the inhibition is not known. It is not a consequence of activation of the omega neuron. Its characteristics are not consistent with those of known inhibitory inputs to the omega neuron.

Animals often must attend to and analyze a single acoustical signal despite the simultaneous presence of other potentially distracting signals. Crickets encounter this "cocktail party problem" (Cherry, 1953) in analyzing the calling song, a speciesspecific signal that is produced by males and subserves several important social functions (Alexander, 1960). Several males may sing simultaneously within earshot of a listening animal (Campbell and Clarke, 1971; Cade, 1981), which nevertheless must analyze individual songs to determine, for example, whether their singers are conspecific. Because many of their auditory neurons can be studied as identified individuals (Casaday and Hoy, 1977; Wohlers and Huber, 1978, 1982), crickets present a favorable system in which to address aspects of this general problem of signal analysis at the cellular level.

Received Sept. 15, 1987; revised Nov. 9, 1987; accepted Nov. 11, 1987.

This work was supported by the Natural Sciences and Engineering Research Council of Canada. I thank Drs. R. Chase and R. M. Robertson for their comments on an earlier version of the manuscript.

Correspondence should be addressed to Dr. Gerald S. Pollack, Department of Biology, McGill University, 1205 Avenue Dr. Penfield, Montreal, Quebec, Canada H3A 1 B1.

Copyright $(\mathcal{C} 1988$ Society for Neuroscience $0270-6474 / 88 / 072635-06 \$ 02.00 / 0$
Experiments on the phonotactic behavior of crickets stimulated with synthetic models of calling song have shown that a crucial cue for species identity is the temporal pattern of the signal (Popov and Shuvalov, 1977; Pollack and Hoy, 1979, 1981; Thorson et al., 1982; Stout et al., 1983; Doolan and Pollack, 1985). However, the overlap of several songs in time and space results in the obfuscation of their temporal structures. That crickets are able to analyze temporal patterns under these conditions has been demonstrated in behavioral experiments, which have shown that crickets can express a clear phonotactic preference for one of 2 simultaneously presented stimuli that differ only in temporal pattern (Pollack and Hoy, 1979, 1981; Pollack, 1982) provided they are sufficiently separated in space (Pollack, 1986). It is likely that crickets accomplish this discrimination by comparing the stimuli on the 2 sides and choosing the appropriate one. Comparison implies that both temporal patterns are represented, independently and simultaneously, in the nervous system. Electrophysiological recordings have shown that a bilateral pair of identified neurons, the omega neurons (interneuron 2 of Casaday and Hoy, 1977; ON1 of Wohlers and Huber, 1982; LSAN of Popov et al., 1978), can encode 2 simultaneous temporal patterns; each omega neuron selectively attends to the stimulus played from its soma-ipsilateral side, even though the soma-contralateral stimulus is effective when it is presented alone (Pollack, 1986). The present paper concerns the physiological mechanism for this selective attention.

\section{Materials and Methods}

Laboratory-reared Teleogryllus oceanicus females were used in all experiments. After removal of the meso- and metathoracic legs and the wings, animals were fixed ventral side uppermost to a platform and the prothoracic legs (on which the ears are found) were flexed at the femorotibial joint and affixed to the sides of the prothorax in a position similar to that adopted during flight. The prothoracic ganglion was exposed by dissecting away the overlying cuticle and was supported on a steel platform. Intracellular recordings were made with Lucifer yellow-filled electrodes [3\% Lucifer yellow CH (Aldrich) dissolved in $0.1 \mathrm{M} \mathrm{LiCl}$, and the identity of the recorded cell was confirmed after each experiment by inspecting its morphology following iontophoresis of dye.

Stimuli were electronically synthesized song models, which were produced by amplitude-modulating a sine wave with an electronic switch. Temporal features of the modulation envelope, stimulus intensity, and carrier frequency could all be varied under computer control. The stimuli were presented by 2 loudspeakers that were placed in the same horizontal plane as the cricket at a distance of $35 \mathrm{~cm}$, orthogonal to the cricket's longitudinal axis. Surfaces and objects in the recording chamber were padded with fiberglass insulating material to reduce echoes.

\section{Results}

\section{Selective response of the omega neuron}

Pollack (1986) described the omega neuron's selective response to the soma-ipsilateral stimulus when 2 stimuli are played simultaneously from the 2 sides. Figure $1, A, B$, illustrates this 


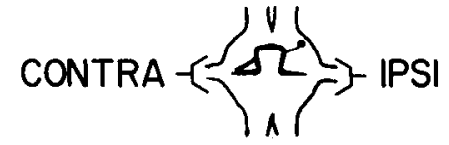

A.

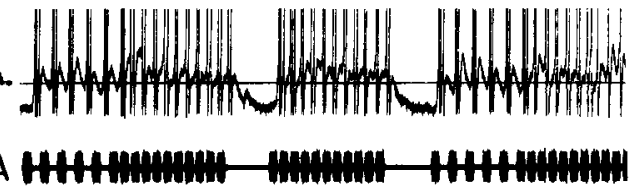

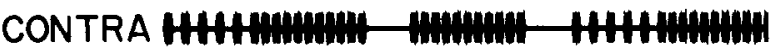

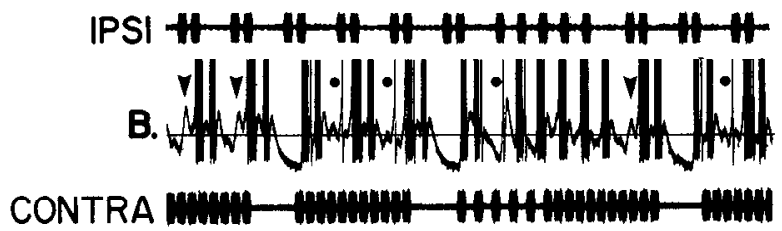

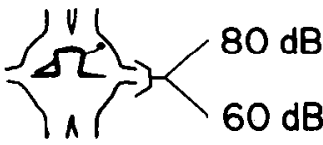
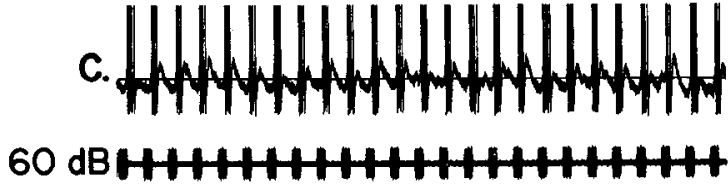

$$
10 \mathrm{mV} \frac{}{500 \mathrm{~ms}}
$$

Figure 1. Responses of the omega neuron to single and dual stimulation. The stimulus temporal patterns are modeled after cricket songs (Pollack and Hoy, 1981). All records begin $5 \mathrm{sec}$ after the commencement of stimulation. $A$, Response to an $80 \mathrm{~dB}$ contralateral stimulus. Each sound pulse elicits a burst of spikes (which are truncated in these records). Between groups of sound pulses the membrane is hyperpolarized with respect to prestimulus membrane potential, which is indicated by the horizontal line superimposed on the record. $B$, Response to simultaneous stimulation from both sides ( $80 \mathrm{~dB}$ for both stimuli). The neuron's response is dominated by the ipsilateral stimulus. The formerly effective contralateral stimulus elicits primarily subthreshold EPSPs (some of which are indicated with arrows); only a few sound pulses result in single action potentials (circles). Between responses, membrane potential is more hyperpolarized than in $A$. $C$ and $D$, Responses when stimuli of differing intensities are presented from the same (soma-ipsilateral) side; the $20 \mathrm{~dB}$ difference in intensity approximates the difference in sensitivity of this preparation to stimulation from the 2 sides. $C$, The $60 \mathrm{~dB}$ stimulus produces a burst of spikes for each sound pulse and modest interburst hyperpolarization when presented alone. $D$, When the $80 \mathrm{~dB}$ stimulus is added, it dominates the neuron's response, and also elicits more profound hyperpolarization. Arrows and circles indicate EPSPs and spikes, respectively, that are elicited by the $60 \mathrm{~dB}$ stimulus.

phenomenon. The neuron responds to a suprathreshold contralateral stimulus with a clear burst of spikes following each sound pulse, so long as the stimulus is played alone (Fig. 1A). When a second stimulus is presented simultaneously from the ipsilateral side, at the same intensity, the neuron's spiking response

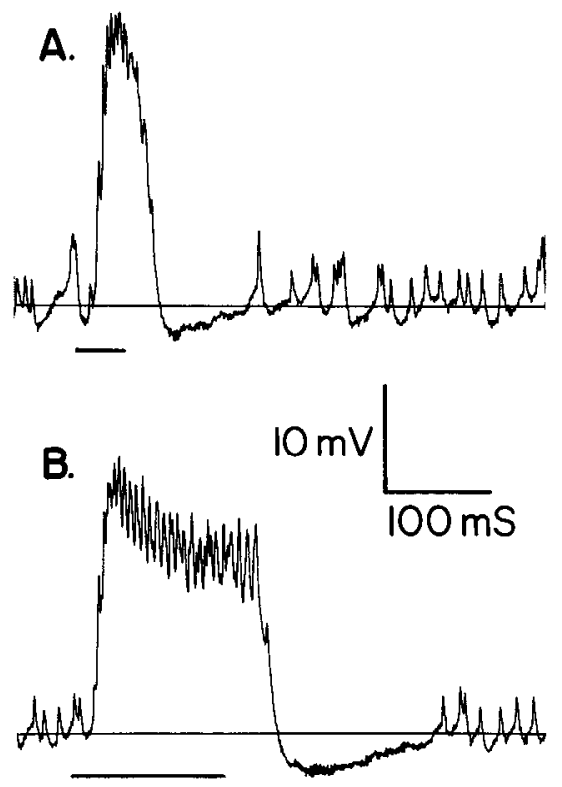

Figure 2. Hyperpolarization depends on stimulus duration. Each trace is an average of 5 responses to a $5 \mathrm{kHz}, 70 \mathrm{~dB}$ tone. Bars beneath traces are stimulus markers; duration is $50 \mathrm{msec}$ in $A, 150 \mathrm{msec}$ in $B$. Horizontal line drawn through trace indicates resting membrane potential. primarily reflects the ipsilateral stimulus (Fig. $1 B$ ); contralateral sound pulses now only occasionally result in single spikes.

Because the auditory system in general, and the omega neuron in particular, is directionally selective (Popov et al., 1978; Wohlers and IIuber, 1982), a contralateral stimulus is in some respects equivalent to a low-intensity ipsilateral stimulus. This intensity difference, which is a consequence of sound direction, is responsible for the neuron's selective response. When lowand high-intensity stimuli are mixed on a single channel and presented from the same (ipsilateral) loudspeaker the omega neuron responds selectively to the more intense of the 2 (Fig.

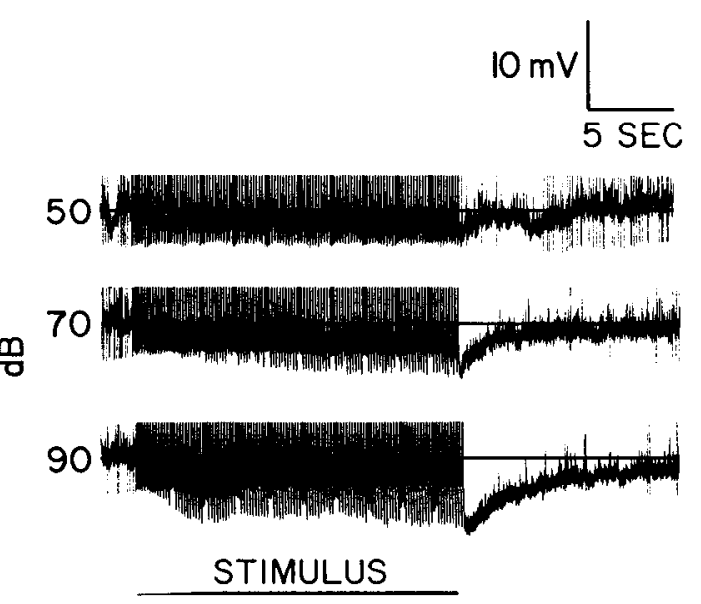

Figure 3. Hyperpolarization is intensity-dependent. The stimulus was a model of the species calling song (Pollack and Hoy, 1981). 
$1 D$ ), even though the low-intensity stimulus elicits a clear response when it is presented alone (Fig. 1C).

\section{Biphasic response of the omega neuron}

In addition to exciting the omega neuron, sound stimuli also cause inhibition, which can be seen as hyperpolarization following the excitatory response. Short, single stimuli elicit brief hyperpolarization of moderate amplitude, while longer stimuli produce more profound and longer-lasting hyperpolarization (Fig. 2). Pronounced inhibition is also elicited by a series of closely spaced shorter tones, such as comprise calling song (Figs. 1,3). In Figure 1 inhibition is apparent as tonic hyperpolarization of the membrane. The time course of the hyperpolarization can be seen in Figure 3, which also demonstrates its strong dependence on stimulus intensity. The inhibitory nature of the hyperpolarization is indicated by 2 concomitant phenomena: (1) during stimulation with a model of the calling song, the number of action potentials elicited by successive sound pulses decreases with a time course approximating that of the buildup of the hyperpolarization (Fig. 4A); and (2) the hyperpolarization is associated with an increase in membrane conductance (Fig. 4R).

The intensity-dependent inhibition serves to restrict the neuron's "attention" to the more intense of the 2 stimuli. The way in which this occurs can be understood by reexamination of Figure 1. Figure 1, $A, C$, shows that the less intense (= contralateral) stimulus, when presented alone, elicits both excitation (bursts of spikes) and inhibition (hyperpolarization of the membrane, between bursts, below the prestimulus level). Because the stimulus is of relatively low intensity, the excitation and associated inhibition are modest. When the more intense (= ipsilateral) stimulus is added (Fig. 1, $B, D$ ), both excitation and inhibition increase; the additional excitation results in bursts of spikes that are temporally correlated with the more intense stimulus, and the additional inhibition causes the interburst membrane potential to dip further below prestimulus level. The strong excitation elicited by the more intense stimulus is sufficient to overcome the summed inhibition resulting from the 2 stimuli, but the relatively weak excitation elicited by the less intense stimulus is not. Consequently, the less intense stimulus generally elicits subthreshold EPSPs while the more intense stimulus is simultaneously present, and its temporal pattern is filtered out of the neuron's spike train.

\section{Inhibition is not a consequence of activation of the omega neuron}

One possible explanation for the inhibitory component of the omega neuron's response is that it is a consequence of activation of the neuron, due either to processes intrinsic to the cell, e.g., a Ca-activated K conductance (Meech, 1978), or to synaptic inhibition from one or more neurons that are driven, directly or otherwise, by the omega neuron. A second possibility is that the inhibitory component of the response is due to parallel circuitry that, like the recorded cell, is driven by the acoustical stimulus. The most straightforward way to discriminate between these alternatives is to drive the omega cell with intracellular current injections and determine whether this stimulus also elicits a biphasic response. Responses to depolarizing current show no inhibitory component. However, I have not been able to inject sufficient current to cause spiking rates as high as those elicited by even relatively low-intensity sound stimuli, so this experiment is, unfortunately, inconclusive. Additional evidence
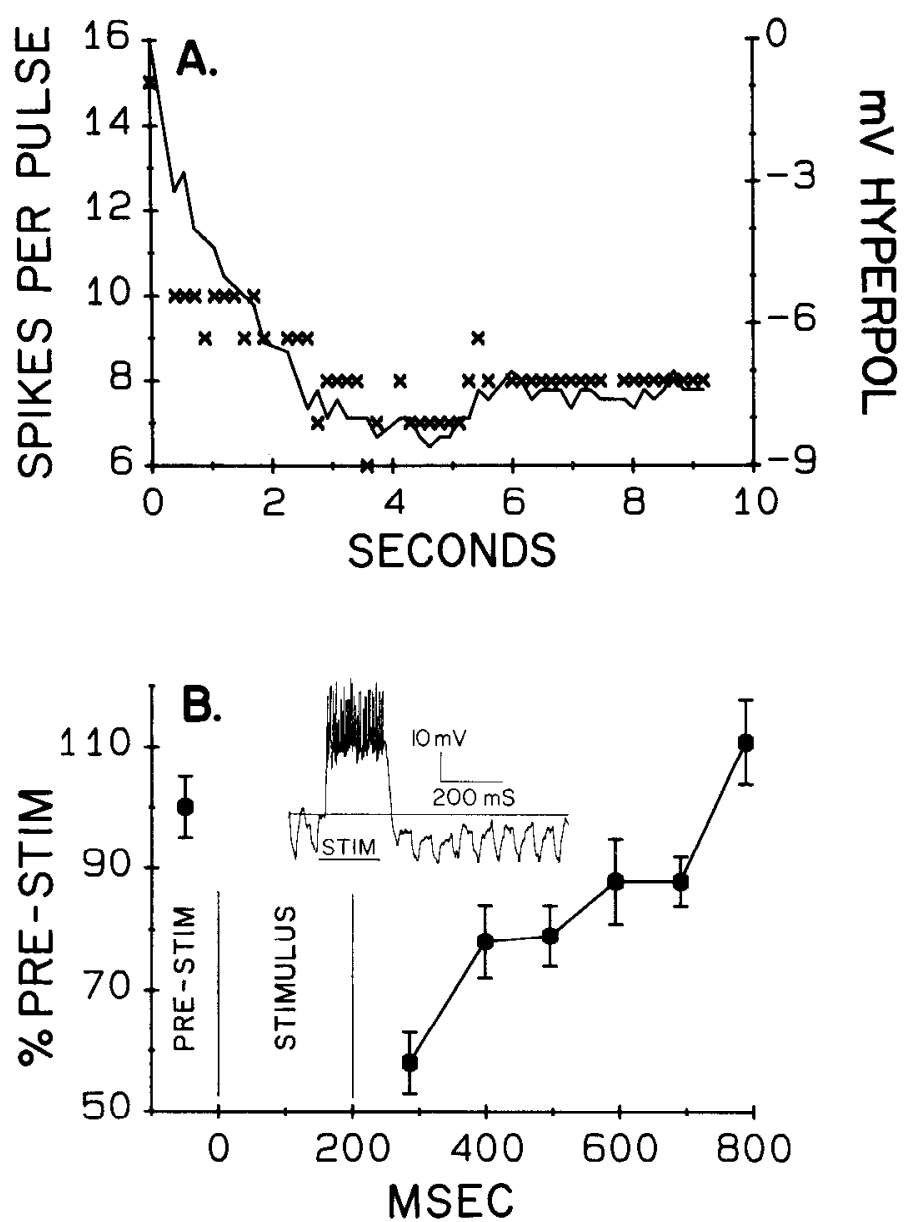

Figure 4. A, Hyperpolarization is associated with decreased spiking response. Data points $(\mathrm{X})$ plot the number of spikes elicited by individual, $30 \mathrm{msec}$ sound pulses comprising the song model. Responses are shown for 50 selected sound pulses of the 115 that occurred during the time included in the graph. [The selected pulses are those that follow the longest $(141 \mathrm{msec})$ interpulse intervals in the stimulus.] Solid line traces the hyperpolarization at the beginning of each of these sound pulses. Stimulus intensity, $90 \mathrm{~dB}$. B. Hyperpolarization is associated with an increase in membrane conductance. The neuron was stimulated with a $200 \mathrm{msec}, 80 \mathrm{~dB}$ tone, which resulted in a prolonged hyperpolarizing tail (horizontal line in inset indicates prestimulus membrane potential). Constant, $-1 \mathrm{nA}$ current pulses were injected through the recording electrode by means of a balanced bridge circuit and resulted in downward deflections (inset). Graph shows mean $\pm \mathrm{SE}(n=6-9)$ deflection amplitudes during the periods of hyperpolarization following 5 successive sound pulses, expressed as the percentage of the mean deflection amplitude for the 5 current pulses that immediately preceded each sound pulse. Poststimulus current pulses were assigned to $100 \mathrm{msec}$ time bins, beginning $240 \mathrm{msec}$ after the onset of sound. The points represent means of deflection amplitudes within each time bin and are plotted at the mean time of occurrence of the current pulses in each bin. During the period of hyperpolarization, the deflections were smaller, indicating an increase in membrane conductance. Stimulus artifacts in inset retouched.

supporting the possibility that the inhibition results from parallel circuits comes from its frequency sensitivity. The omega neuron can be excited over a wide frequency range and shows sensitivity peaks near $5 \mathrm{kHz}$, which is near the carrier frequency of intraspecific signals (Leroy, 1964), and in the ultrasound (Atkins and Pollack, 1986). Figure 5 shows, however, that inhibition is more effectively elicited by $5 \mathrm{kHz}$ than by ultrasound, even though the 2 stimuli may excite the cell equally well. 
A.
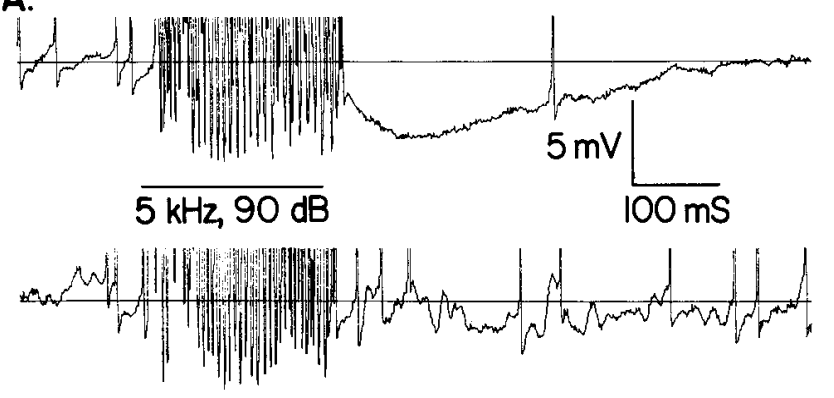

$25 \mathrm{kHz}, 90 \mathrm{~dB}$

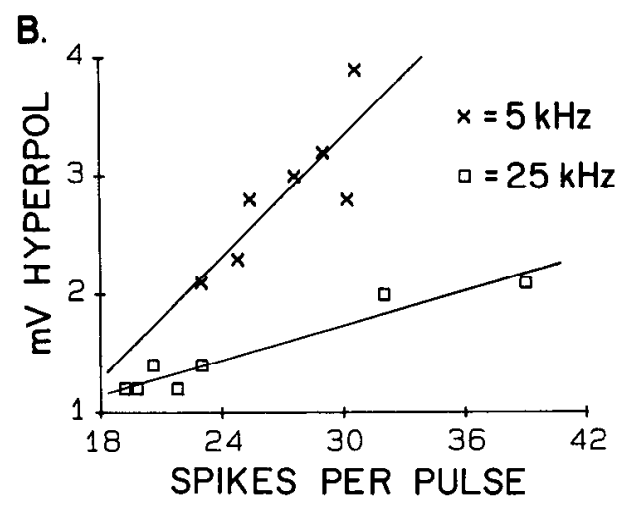

Figure 5. Frequency specificity of inhibition. A, Strong hyperpolarization is elicited by a $90 \mathrm{~dB}, 5 \mathrm{kHz}$ tone (trace shows average of 5 responses) but not by a $90 \mathrm{~dB}, 25 \mathrm{kHz}$ tone (average of 4 responses). The stimuli were of nearly equal effectiveness in eliciting excitation, as judged by spike counts: $5 \mathrm{kHz}, 32.8 \pm 2.4$ spikes/response (mean \pm SD); $25 \mathrm{kHz}, 35.6 \pm 0.5$. $B$, From another preparation. Tones $(200$ msec) with carrier frequency of 5 or $25 \mathrm{kHz}$ were played at a number of intensities (from $60-90 \mathrm{~dB}$ for $5 \mathrm{kHz}, 70-100 \mathrm{~dB}$ for $25 \mathrm{kHz}$ ). For each intensity-frequency combination the mean number of spikes per response and the mean maximum postresponse hyperpolarization were calculated for 5 successive responses. The graph shows that, although hyperpolarization was produced at $25 \mathrm{kHz}$, hyperpolarization was more pronounced for $5 \mathrm{kHz}$ stimuli at equivalent levels of excitation (indicated by spike count). The lines shown were determined by least-squares regression analysis.

\section{Discussion}

\section{Nature and source of inhibition}

The selective response of the omega neuron to the more intense (or ipsilateral) of 2 stimuli is ascribed here to a slow inhibitory synaptic potential, which serves to dampen the cell's response so that only the more intense of the 2 stimuli remains suprathreshold. Because it is intensity dependent, the inhibition acts as an "automatic gain control," i.e., it adjusts the neuron's output in accordance with its input. This aspect of the inhibition permits the neuron to respond selectively to the more intense stimulus over a wide range of absolute intensities. Pollack (1986) reported that the omega neuron can respond selectively to the ipsilateral stimulus, when an equally intense stimulus is presented contralaterally, at least over the intensity range of 60 $90 \mathrm{~dB}$. Although it is clear that the postsynaptic inhibition described here contributes to the neuron's selective response, it has not been established that it is the only factor responsible for selective attention. It is possible, for example, that side- selective presynaptic inhibition of inputs to the omega cell also occurs.

Inhibition is best elicited by prolonged stimuli with relatively low carrier frequency (Figs. 2, 3). These properties permit establishment of inhibition during stimulation with calling song, which is both long-lasting and of low carrier frequency, but not in response to brief, occasional sounds. Consequently, inappropriate damping of the neuron's responsiveness, and the attendant potential loss of vigilance, is avoided. The match between the spectral and temporal requirements for inhibition and the structure of the calling song lends support to the notion that the inhibition is a specialization for intraspecific communication.

The omega neuron is known to recieve inhibitory input from 2 sources; substrate vibration receptors on the ipsilateral foreleg (Wiese, 1981) and the contralateral omega neuron (Selverston et al., 1985). Neither of these is likely to account for the inhibition underlying selective attention. Vibration input is unlikely to be important because the stand that supported the cricket was mechanically isolated from the loudspeaker supports, rendering significant transmission of substrate vibration to the cricket unlikely, and because the sound frequency that evoked inhibition in the present experiments, $5 \mathrm{kHz}$, is well above the range of best sensitivity of the vibration receptors-ca. 0.7-1.0 $\mathrm{kHz}$ (Dambach, 1972). The contralateral omega neuron is an unlikely candidate for the inhibition described here for several reasons. First, it produces short-latency, short-duration IPSPs (Sclverston ct al., 1985), while the inhibition responsible for selective attention is more tonic in character and has build-up and decay times of up to several seconds (Figs. 3 and $4 A$ ). Second, the frequency sensitivity of the inhibition (Fig. 5) is not consistent with its resulting from activity in the contralateral omega neuron. The binaural difference in sensitivity of the omega neuron for $25 \mathrm{kHz}$ stimuli is similar to, and perhaps slightly less than, that for $5 \mathrm{kH} /$ stimuli (G. S. Pollack, unpublished observations). [One might expect the binaural difference to be greater for $25 \mathrm{kHz}$ because, due to its shorter wavelength, it would be more effectively shadowed by the cricket's body. However, special acoustical mechanisms exist that accentuate the binaural difference at $5 \mathrm{kHz}$ (Hill and Boyan, 1977).] Consequently, the contralateral omega neuron is stimulated at least as strongly, relative to the ipsilateral neuron, by a $25 \mathrm{kHz}$ stimulus as by a $5 \mathrm{kHz}$ stimulus, and any inhibitory influence that it exerts on the ipsilateral cell should also be at least as powerful at the higher frequency. Finally, Pollack (1986) found no difference in the fidelity with which the ipsilateral temporal pattern was encoded, while a contralateral stimulus was presented simultaneously, following contralateral deafening. If the contralateral omega neuron were the major source of the inhibition that permits selective encoding of the ipsilateral stimulus, then contralateral deafening would be expected to have a pronounced detrimental effect on selective encoding. For the present, the source of the inhibition described here remains unknown. Its slow time course raises the possibility that it may be due to the action of a neuromodulatory substance, rather than to classical synaptic inhibition.

\section{Comparison with selective attention in vertebrates}

The attentional mechanism described here depends on physical characteristics of the signal (frequency, intensity, duration) to restrict the encoding of information at an early stage of processing [the omega neuron is believed to be directly postsynaptic to auditory receptor cells (Wohlers and Huber, 1985)] and is 
analogous to the "stimulus set selection" that has been proposed to contribute to attention in humans (Broadbent, 1971). A crucial difference between stimulus set selection and the phenomenon investigated in the present study is that the former is under volitional control (one can choose the stimulus set, i.e., the constellation of physical features of the stimulus, to which one will attend), while the latter presumably is not. Nevertheless, the result of this early selection in crickets is the same as that hypothesized in humans, namely, the production of a "clean" copy of the stimulus, with irrelevant features removed, that can be used in later stages of analysis. In crickets, further analysis of stimulus temporal pattern occurs in the brain (Schildeberger, 1984), and it is, presumably, here that it is decided whether the pattern is that of a conspecific, and therefore whether the signal is an appropriate object of a behavioral response. The result of this later analysis can then direct the attention of the animal as a whole towards the stimulus, resulting in the behavioral response of phonotaxis. This hierarchical model of attention is similar to that which has been proposed for humans, where increasingly sophisticated analyses are thought to occur at successively later stages of processing (Hillyard, 1985).

\section{References}

Alexander, R. D. (1960) Sound communication in Orthoptera and Cicadidae. In Animal Sounds and Communication, W. E. Lanyon and W. N. Tavolga, eds., pp. 38-92, American Institute of Biological Sciences, Washington, D.C.

Atkins, G., and G. S. Pollack (1986) Age dependent occurrence of an ascending axon on the omega neuron of the cricket, Teleogryllus oceanicus. J. Comp. Neurol. 243: 527-534.

Broadbent, D. R. (1971) Decision and Stress, Academic, New York.

Cade, W. H. (1981) Field cricket spacing, and the phonotaxis of crickets and parasitoid flies to clumped and isolated cricket songs. $Z$. Tierpsychol. 55: 365-375.

Campbell, D. J., and D. J. Clarke (1971) Nearest neighbour tests of significance for non-randomness in the spatial distribution of singing crickets (Teleogryllus commodus (Walker)). Anim. Behav. 19: 750756.

Casaday, G. B., and R. R. Hoy (1977) Auditory interneurons in the cricket Teleogryllus oceanicus: Physiological and anatomical properties. J. Comp. Physiol. 121: 1-13.

Cherry, E. C. (1953) Some experiments on the recognition of speech, with one and with two ears. J. Acoust. Soc. Am. 25: 975-979.

Dambach, M. (1972) Der Vibrationssinn der Grillen. I. Schwellenmessungen an Beinen frei beweglicher Tiere. J. Comp. Physiol. 79: 281-304.

Doolan, J. M., and G. S. Pollack (1985) Phonotactic specificity of the cricket Teleogryllus oceanicus: Intensity-dependent selectivity for temporal parameters of the stimulus. J. Comp. Physiol. 157: 223233.
Hill, K. G., and G. S. Boyan (1977) Sensitivity to frequency and direction of sound in the auditory system of crickets (Gryllidae). J. Comp. Physiol. 121: 79-97.

Hillyard, S. A. (1985) Electrophysiology of human selective attention. Trends Neurosci. 8: 400-405.

Leroy, Y. (1964) Transmission du paramètre fréquence dans le signal acoustique des hybrides Fl et $\mathrm{P}$ x Fl de deux grillons: $T$. commodus Walker et T. oceanicus Le Guillou (Orthoptères, Ensifêres). CR Acad. Sci. Paris [D] 259: 892-895.

Meech, R. W. (1978) Calcium-dependent potassium activation in nervous tissues. Annu. Rev. Biophys. Bioeng. 7: 1-18.

Pollack, G. S. (1982) Sexual differences in cricket calling song recognition. J. Comp. Physiol. 146: 217-221.

Pollack, G. S. (1986) Discrimination of calling song models by the cricket, Teleogryllus oceanicus: The influence of sound direction on neural encoding of the stimulus temporal pattern and on phonotactic behavior. J. Comp. Physiol. 158: 549-561.

Pollack, G. S., and R. R. Hoy (1979) Temporal pattern as a cue for species-specific calling song recognition in crickets. Science 204: 429432.

Pollack, G. S., and R. R. Hoy (1981) Phonotaxis to individual rhythmic components of a complex cricket calling song. J. Comp. Physiol. 144: $367-373$.

Popov, A. V., and V. F. Shuvalov (1977) Phonotactic behavior of crickets. J. Comp. Physiol. 119: 111-126.

Popov, A. V., A. M. Markovich, and A. S. Andjan (1978) Auditory interneurons in the prothoracic ganglion of the cricket, Gryllus bimaculatus de Geer. J. Comp. Physiol. 126: 183-192.

Schildeberger, K. (1984) Temporal selectivity of identified auditory neurons in the cricket brain. J. Comp. Physiol. 155: 171-185.

Selverston, A. I., H.-U. Kleindienst, and F. Huber (1985) Synaptic connectivity between cricket auditory interneurons as studied by selective photoinactivation. J. Neurosci. 5: 1283-1292.

Stout, J. F., C. H. Dehaan, and R. W. McGhee (1983) Attractiveness of the male Acheta domesticus calling song to females. I. Dependence on each of the calling song features. J. Comp. Physiol. 153: 509-521.

Thorson, J., T. Weber, and F. Huber (1982) Auditory behavior of the cricket. II. Simplicity of calling-song recognition in Gryllus, and anomalous phonotaxis at abnormal carrier frequencies. J. Comp. Physiol. 146: 361-378.

Wiese, K. (1981) Influence of vibration on cricket hearing: Interaction of low frequency vibration and acoustic stimuli in the omega neuron. J. Comp. Physiol. 143: 135-142.

Wohlers, D. W., and F. Huber (1978) Intracellular recording and staining of cricket auditory interneurons (Gryllus campestris L., Gryllus bimaculatus de Geer). J. Comp. Physiol. 127: 11-28.

Wohlers, D. W., and F. Huber (1982) Processing of sound by six types of neurons in the prothoracic ganglion of the cricket, Gryllus campestris L. J. Comp. Physiol. 146: 161-173.

Wohlers, D. W., and F. Huber (1985) Topographical organization of the auditory pathway within the prothoracic ganglion of the cricket Gryllus campestris L. Cell Tissue Res. 239: 555-565. 